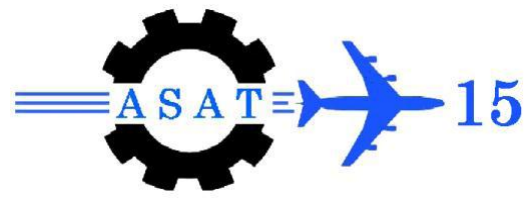

\title{
Development of a New Electronically Controlled Torque Vectoring Device for All-Wheel-Drive Vehicles
}

\author{
E. S. Sheha* , A. M. Sharaf ${ }^{\dagger}$, Y. H. Hossameldeen ${ }^{\star}$ and A. M. Helmy ${ }^{\S}$
}

\begin{abstract}
This paper presents a comprehensive torque distribution device to actively control the torque distribution across the output shafts of an ordinary open differential. The proposed device can be used to control both the magnitude and direction of the torque between the driving axles of all-wheel-drive (AWD) vehicles and / or between the driving wheels of its rear axle. Both the design procedures and the development considerations of the device are outlined. Furthermore, a dedicated drive-train test rig is devised in order to exemplify and measure the characteristics of the controlled torque. The drivetrain test rig comprises different sensors such as wireless torque and driving speed measurements in addition to an electronic control module which regulates both the torque magnitude and direction. The experimental results illustrate the possibilities to bias variable amount of torque across the output shafts of open differential and therefore improve the traction potential during excessive tire slip conditions.
\end{abstract}

Keywords: Active differential, torque vectoring, torque distribution devices.

\section{Nomenclature}

$b_{1}, b_{2} \quad$ Damping rates of the input and output shafts [N.m.s/rad]

$F \quad \quad$ Normal force applied to the clutch plates [N]

$I_{1}, I_{2} \quad$ Moments of inertia of the input and output shafts

$n \quad$ Number of plates [--]

$R \quad$ Road turning radius [m]

$r \quad$ Tire rolling radius $[\mathrm{m}]$

$r_{1}, r_{2}$ The inner and outer radii $\left(r_{1}\right)$ and $\left(r_{2}\right)$ respectively, [m]

$r_{e} \quad$ The effective radius [m]

$T_{F} \quad$ The clutch frictional torque [N.m]

$T_{F \max } \quad$ Maximum torque capacity which is transmitted by a multi-plate clutch pack

$T_{\text {in }} \quad$ Input Torque [N.m]

$T_{L}, T_{R} \quad$ Measured Torque at Left and right half-shafts respectively [N.m]

$T_{\mu_{L}}, T_{\mu_{R}} \quad$ Applied torque of the input and output shafts [N.m]

\footnotetext{
*Egyptian Armed Forces, Egypt, mtces39@gmail.com

${ }^{\dagger}$ Egyptian Armed Forces, Egypt, al_hossein2005@hotmail.com

${ }^{\ddagger}$ Professor, Chairman of Dept. of Mechanical and Mechatronics Eng., HTI, yehia.hendawy@hti.edu.eg

${ }^{\S}$ Egyptian Armed Forces, Egypt, phd_student 2006@yahoo.com
} 


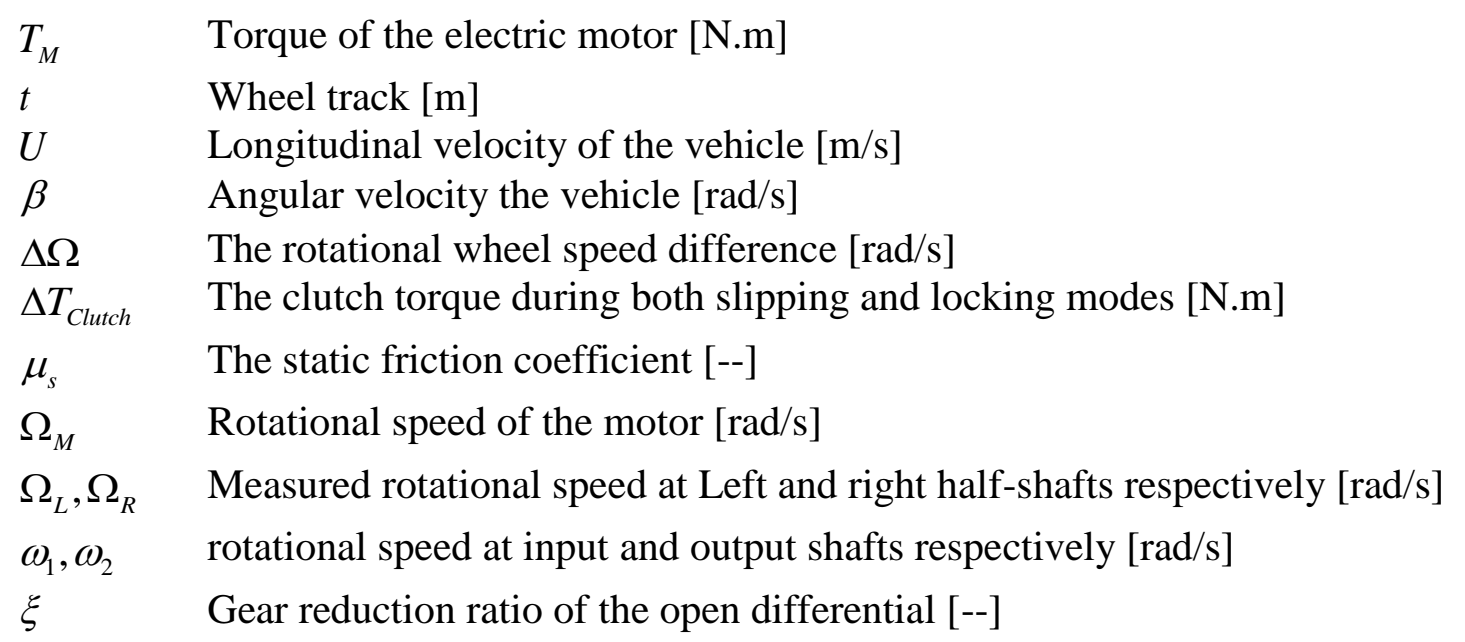

\section{Introduction}

Since it was invented in 1827 by Onésiphore Pecqueur, the conventional open differential has been the standard device for automotive powertrains. This device is simple and effective in providing the necessary speed difference between the driving wheels during vehicle turning. However, because of its inherent equal-torque characteristics, the open differential cannot take full advantage of the available traction at the driving wheels in $\mu$-split or during off-road conditions [1]. To improve the traction potential of an open differential, the fully-locked differential has been introduced where both the wheels rotate at the same speed while the torque is distributed according to the available road adhesion. The drawback of the fullylocked differential arises while driving over rigid roads where a severe transmission windup occurs in addition to an undesirable oversteer tendency at high speed cornering maneuvers $[2]$.

Among the open and fully-locked differentials, several torque distribution devices have been developed over the years varying from passive limited slip differentials to active controllable differentials [3-5]. The passive limited slip differential (LSD) transfers torque across an axle in response to torque input which is called torque-sensitive LSD or wheel driving speed difference which is called speed-sensitive LSD. However, LSD needs to be tuned either to improve traction or to maintain both the handling and stability requirements [6]. This disadvantage has been overcome by introducing electronically controlled differentials that allow the magnitude of torque transferred to be adapted over wide range of operation. Thus, they eliminate the negative impact on handling and providing greater compatibility with ABS and ESP systems [7-9].

Recently, another improvement has been offered by introducing torque vectoring differentials which are widely applied due to its advantage to improve both vehicle traction and directional stability [10-12]. It should be noted that, while active differentials control the magnitude of torque to the side with better traction conditions, torque vectoring differentials provide the total control of both magnitude and direction of the torque transferred. A basic comparison between active controllable differential, torque vectoring differentials and brake control torque vectoring system is shown in Fig. 1.

The majority of the current design solutions for electronically torque-biased devices, used in AWD applications, are based on wet-plate clutches. The amount of torque transferred can be varied by modifying the number of friction plates engaged or by modulating the applied 
pressure. Several mechanisms are used for generating and controlling the applied pressure including ball-ramp arrangements, gear-pumps, axial pistons/cam plates, hydraulic valves, electric motor and screw with nut mechanism,... etc. [13-14].

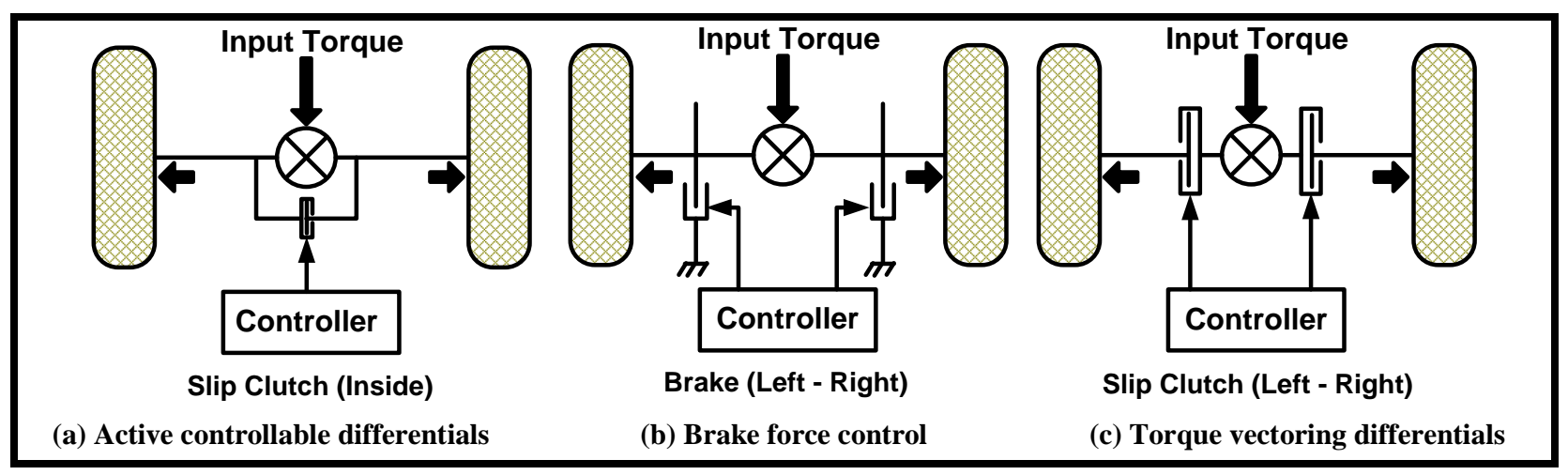

Fig. 1. Modern Torque Controlling Concepts [15]

Based on brake-based torque vectoring approach as illustrated in Fig. 1-b, this paper presents a new torque distribution device for controlling the driving axle's torque whenever one of its wheels slips excessively. Based on direct measurements of the wheels driving speed, a control module regulates the applied force to a set of multi-plate clutches. This mechanism is applied for the faster wheel and thus more torque is biased to the slower one. As a result, the overall traction potential is increased particularly during off-road or $\mu$-split driving conditions.

\section{Design and Development of the Device}

The proposed device comprises a wet-type multi-plate clutch assembly in which the innerplate clutches are connected to a splined shaft while the outer-plate clutches are connected to splined stationary housing as shown in Figs. 2 and 3. The splined shaft is connected to the left half-shaft of the driving axle through a pair of spur gear reduction unit. For the purpose of generating and controlling the magnitude of a clutch assembly engagement force, the device includes a DC motor and screw mechanism. The thrust force is generated by a torque transfer mechanism and then regulated by a control module.

Assuming that the axial force applied to the clutch plates generates a uniform pressure, the maximum torque capacity which is transmitted by a multi-plate clutch pack $\left(T_{F \max }\right)$ can be computed based on the Coulomb friction law. The magnitude of this torque is limited by the normal force applied to the clutch plates $(F)$, the inner and outer radii $\left(r_{1}\right)$ and $\left(r_{2}\right)$ respectively, number of plates $(n)$ and the static friction coefficient $\left(\mu_{s}\right)$ as follows:

$$
T_{F \max }=F \cdot \underbrace{\left(\frac{2}{3} \cdot \frac{r_{2}^{3}-r_{1}^{3}}{r_{2}^{2}-r_{1}^{2}}\right)}_{\text {Effective radius } r_{e}} \cdot n \cdot \mu_{s}=F \cdot r_{e} \cdot n \cdot \mu_{s}
$$




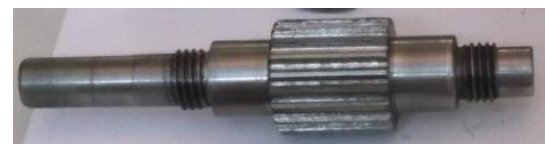

(a) Splined shaft

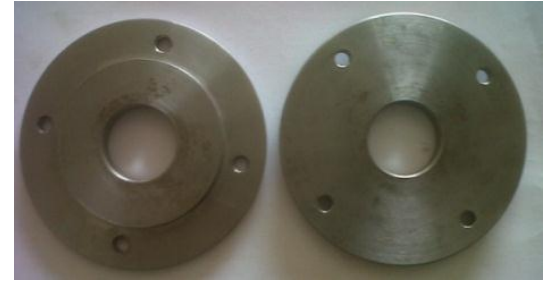

(d) Left and right covers

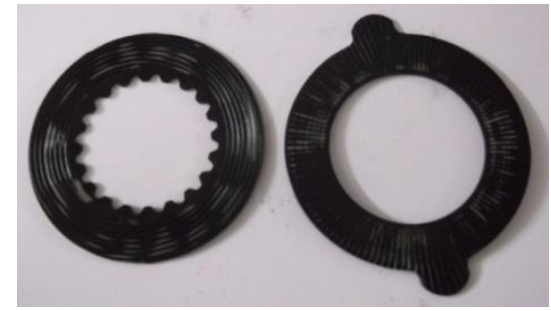

(g) Multi-plate clutches

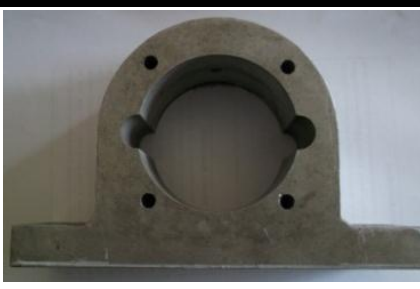

(b) Actuator body

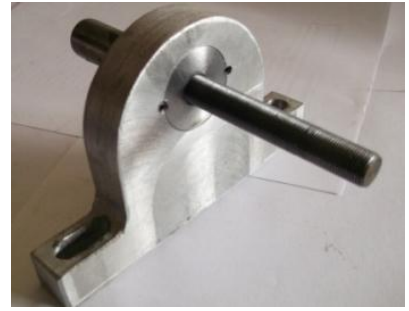

(e) Power screw

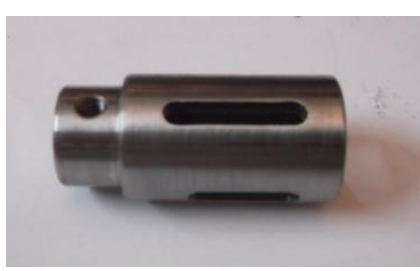

(h) Actuator coupling

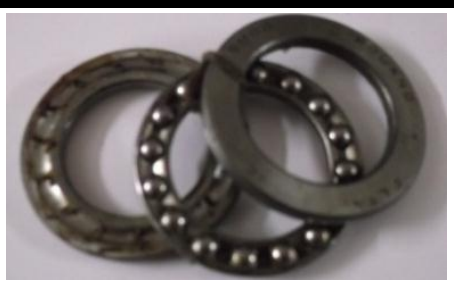

(c) Thrust bearing

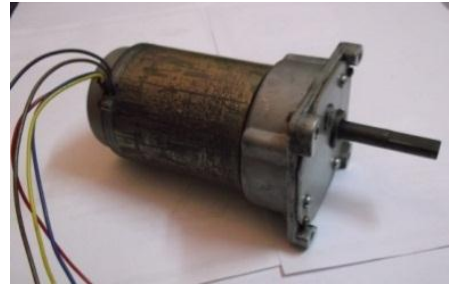

(f) DC motor

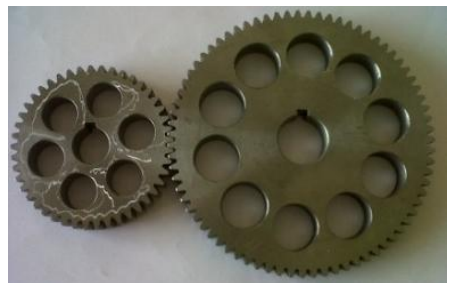

(i) Spur gear reduction

Fig. 2. Typical Components of the Proposed Device

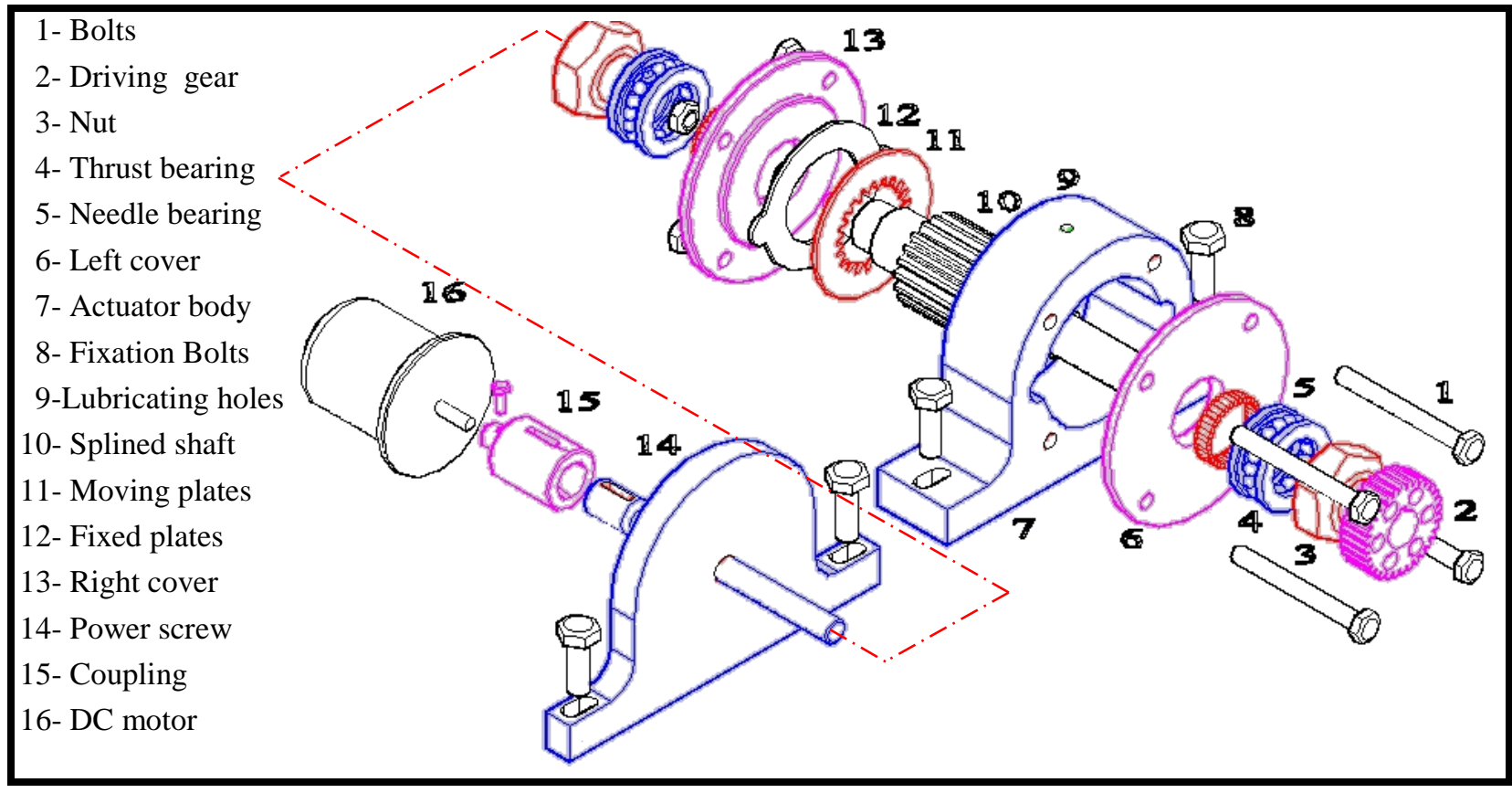

Fig. 3. Three-Dimension Assembly Drawing of the Proposed Device

The clutch torque is calculated during two distinct modes of operation: slipping, where the two plate-sets have differing angular velocities; and lockup, where the two plate-sets rotate together, see Fig. 4. The clutch torque during both slipping and locking modes are given as follows: 


$$
\Delta T_{\text {Clutch }}= \begin{cases}T_{F \max } \cdot \operatorname{sgn}\left(\omega_{1}-\omega_{2}\right) & \left.\rightarrow \text { for } \omega_{1} \neq \omega_{2} \text { (Slipping plates }\right) \\ T_{F} & \left.\rightarrow \text { for } \omega_{1}=\omega_{2} \text { (Locking plates }\right)\end{cases}
$$

Considering the moments of inertia $\left(I_{1}, I_{2}\right)$ and damping rates $\left(b_{1}, b_{2}\right)$ in $(N \cdot \mathrm{m} \cdot \mathrm{s} / \mathrm{rad})$, the equations of motion dynamics during both slipping and locking modes are given as follows:

$$
\left.\begin{array}{l}
I_{1} \cdot \dot{\omega}_{1}=T_{\text {in }}-b_{1} \cdot \omega_{1}-\Delta T_{\text {Clutch }} \\
I_{2} \cdot \dot{\omega}_{2}=\Delta T_{\text {Clutch }}-b_{2} \cdot \omega_{2} \\
\left(I_{1}+I_{2}\right) \cdot \dot{\omega}=T_{\text {in }}-\left(b_{1}+b_{2}\right) \cdot \omega \\
\Delta T_{\text {Clutch }}=T_{f}=\frac{I_{2} \cdot T_{\text {in }}-\left(I_{2} \cdot b_{1}-I_{1} \cdot b_{2}\right) \cdot \omega}{\left(I_{1}+I_{2}\right)}
\end{array}\right\} \rightarrow \text { for } \omega_{1} \neq \omega_{2}
$$

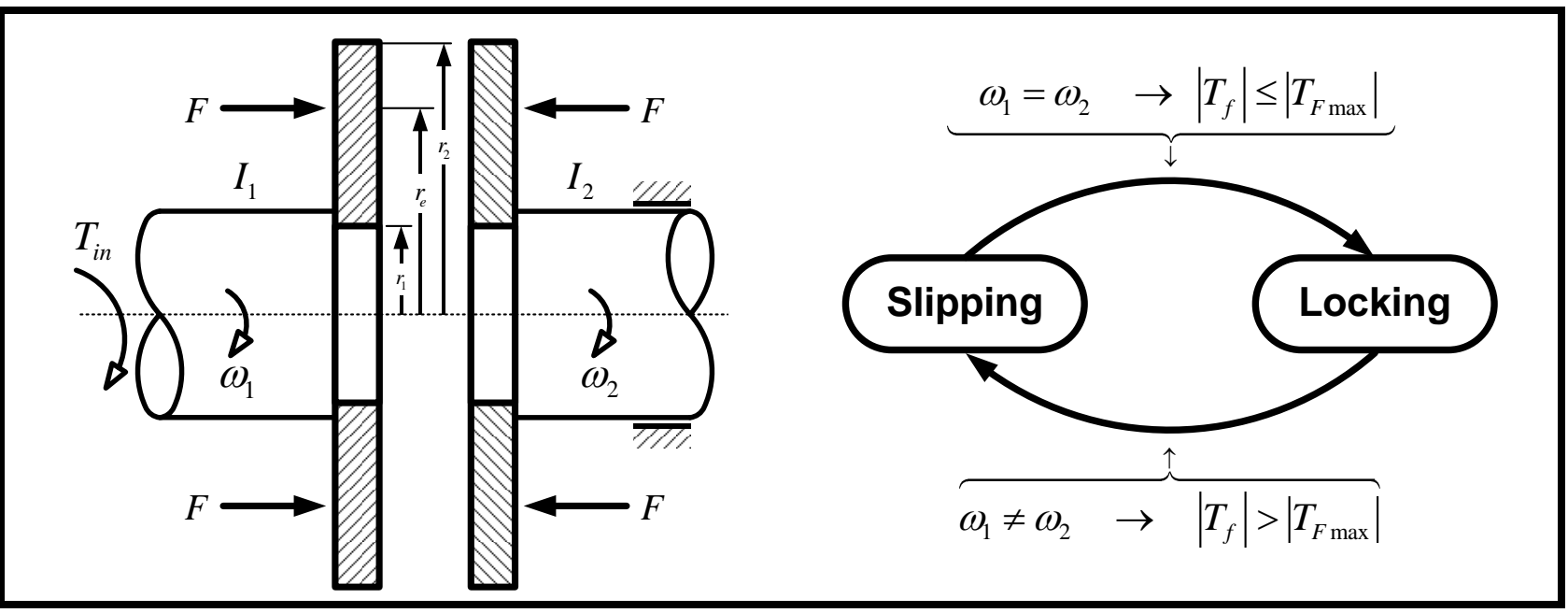

Fig. 4. The Lumped-Parameter Model for the Clutch System

\section{The Test Rig}

In order to investigate the applicability and measure the control characteristics of the proposed torque vectoring device, a special test rig is designed and developed as shown in Fig. 5-a. A full rear drive axle is employed including an open differential, drive half-shafts, brakes and wheels. Some sections from the axle tube are removed such that it is possible to connect the torque vectoring device with the half-shaft through a pair of spur gear reduction unit. In addition, the possibility to measure the driving torque and speed is provided. The axle is driven by a Cardan shaft which is connected from one side to an AC motor of rated output power $7.5 \mathrm{~kW} @ 2900 \mathrm{rpm}$ and from the other side to the axle differential. A speed controller is employed to control the running speed of the AC motor. In addition, two universal joints and flexible spider rubber coupling are introduced to eliminate the torsional vibrations in the drivetrain. 


\subsection{The measuring instruments}

The driving power at each half-shaft is measured in terms of rotational speed and driving torque. A G18-3B2PA photoelectric speed sensor is used to measure the rotational speed for left and right half-shafts in (rpm), see Fig. 5-b. For the measurement of the driving torque applied to both left and right half-shafts, four strain gauges (Biaxial, $120 \mathrm{ohm}, 10 \mathrm{volt}$ excitation) are bonded to each driving axle, see Fig. 5-e. The strain gauges are connected together $(2 \times 2)$ to make $45^{\circ}$ with the force line and $90^{\circ}$ to each other. A Wheatstone bridge method is used to connect the gauges to give four terminals, two of them for excitation and the other for output reading. This configuration achieves strain compensation for axial, bending stress and temperature effect. A wireless module is employed to transmit the measured torque signal as shown in Fig. 5-c. This module consists of the transmitter unit which is fixed to the driving shafts as shown in Fig. 5-d and the receiver unit which is connected to the PC.

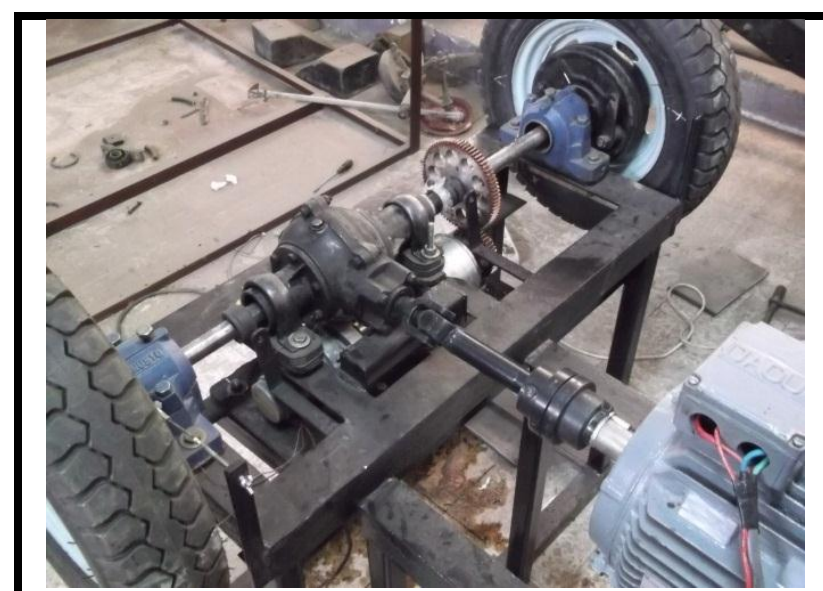

(a) Test rig overall view

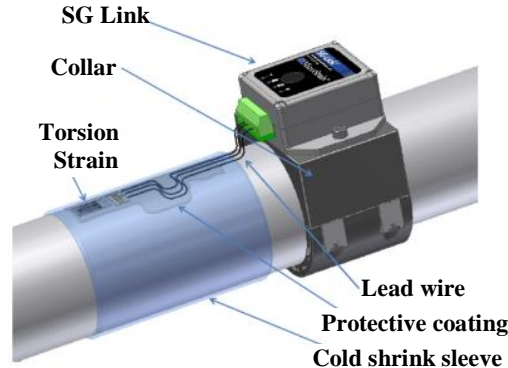

(c) Typical installation set-up

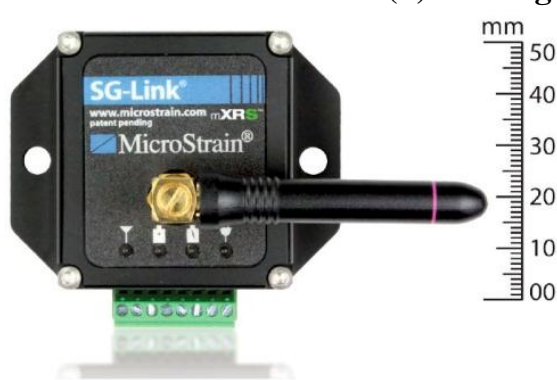

(d) 2-Channel Microstrain SG-Link

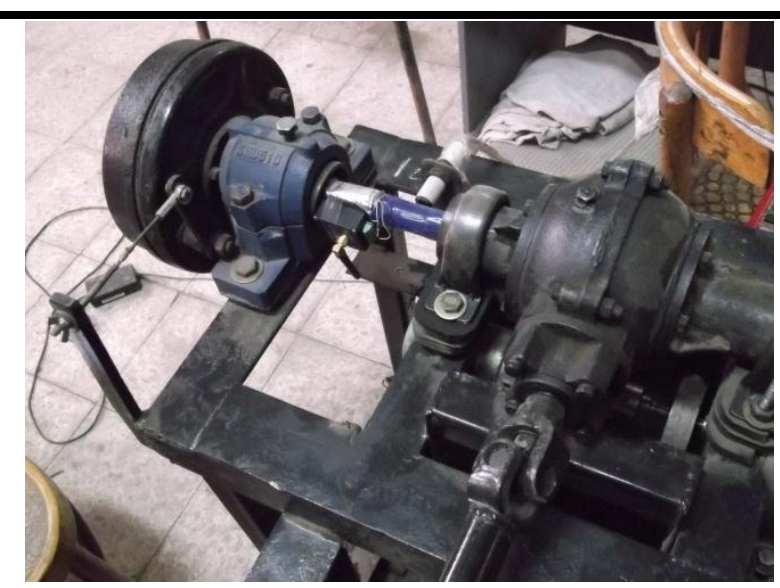

(b) Test rig instrumentation

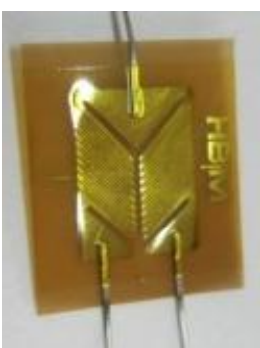

(e) 3/120 XY 21 bi-axial strain gauge

Fig. 5. The Test Rig and Measuring Instruments

\subsection{Signals calibration and acquisition}

\subsubsection{Strain gauge calibration}

The calibration process involves the convergence of the electrical output voltage signal from the strain gauge into its equivalent value of the torque applied to the shafts. Two steps are considered; the first is carried out through the software of the MicroStrain wireless module which converts the strain gauge output voltage to a strain. The second step is completed by converting the measured strain into torque. For this purpose, several weights are applied to a torque arm of $71.5 \mathrm{~cm}$ long as shown in Fig. 6. For each applied weight, the corresponding output strain value is measured and therefore, the relationship 
between the torque and strain for both right and left half-shafts are plotted as shown in Fig. 7. It can be noted that there is a considerable difference in the results for both halfshafts due to the differences in their lengths and consequently they exhibits different strain and torque output.

\subsubsection{Strain gauge zero stability test}

In practice, the strain measurements rarely involve quantities larger than few millistrains $\left(\varepsilon \times 10^{-3}\right)$. Therefore, measuring the strain requires an accurate measurement of very small changes in resistance. To measure such small changes in resistance, strain gauges are always used in a bridge configuration. The general Wheatstone bridge consists of four resistive arms of $120 \Omega$ with an excitation voltage (3v) that is applied across the bridge. The bridge is said to be balanced under any conditions whenever its output voltage is zero. Any change in resistance in any arm of the bridge will result in a non-zero output voltage. The stability test involves adding a fixed resistance of $120 \mathrm{ohm}$ in parallel to one branch of the bridge in order to get a non-zero output. The ideal result is such that, after removing the external resistance, the output voltage should return to its stable zero output as shown in Fig. 8. To ensure the stability of the results, the test is repeated using different resistances of values $350 \Omega$ and $1000 \Omega$.

\subsubsection{System hysteresis test}

This test determines the measured system hysteresis during both loading and unloading directions. Several weights of (5-10-15-20-25) $\mathrm{kg}$ are gradually applied to the moment arm then are removed in a gradual manner. The resultant strain at each step is recorded as shown in Fig. 9. It is clear that, the strain returns to its initial value after adding and removing a given additional weight. Weight, also it is observed that due to weight swinging, some noise occurs in the unloading of the 25 and $20 \mathrm{~kg}$.

\subsubsection{Signal conditioning and data acquisition system}

The output voltage from Wheatstone bridge having four strain gauges is amplified and converted from analog to digital signal. This signal is then transmitted wireless to the node which in turn carries out further processing to obtain the torque value. For the rotational speed measurements, a USB data acquisition (NI USB-6221 DAQ) is employed. In addition, a user-friendly hardware interface is developed using LabVIEW "Laboratory Virtual Instrumentation Engineering Workbench" package and used to modulate the applying normal force to the friction clutches. 


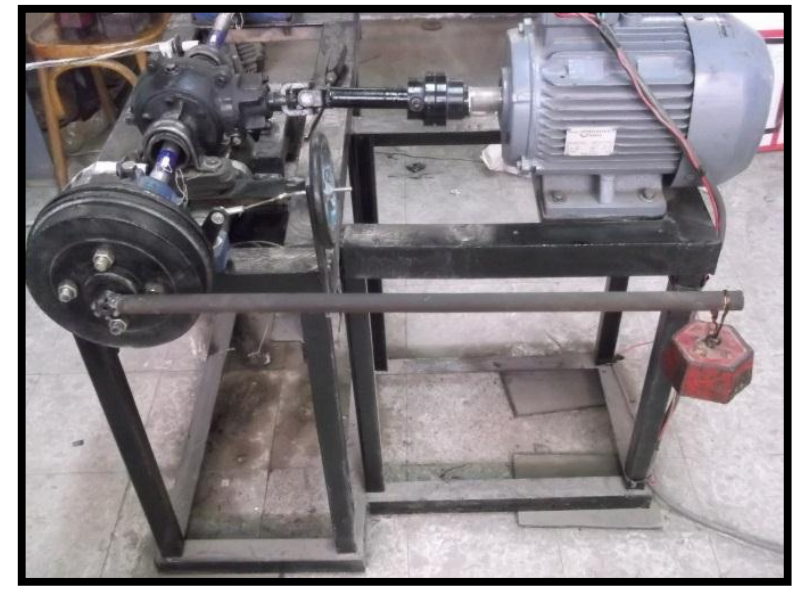

Fig. 6. Experimental Setup for Calibration

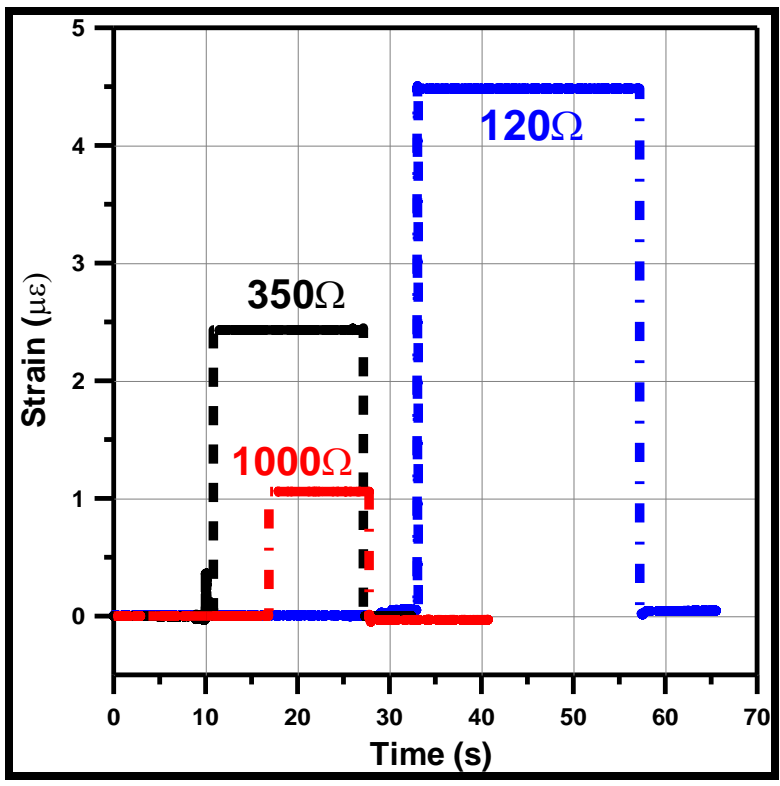

Fig. 8. Strain Gauge Zero Stability Test

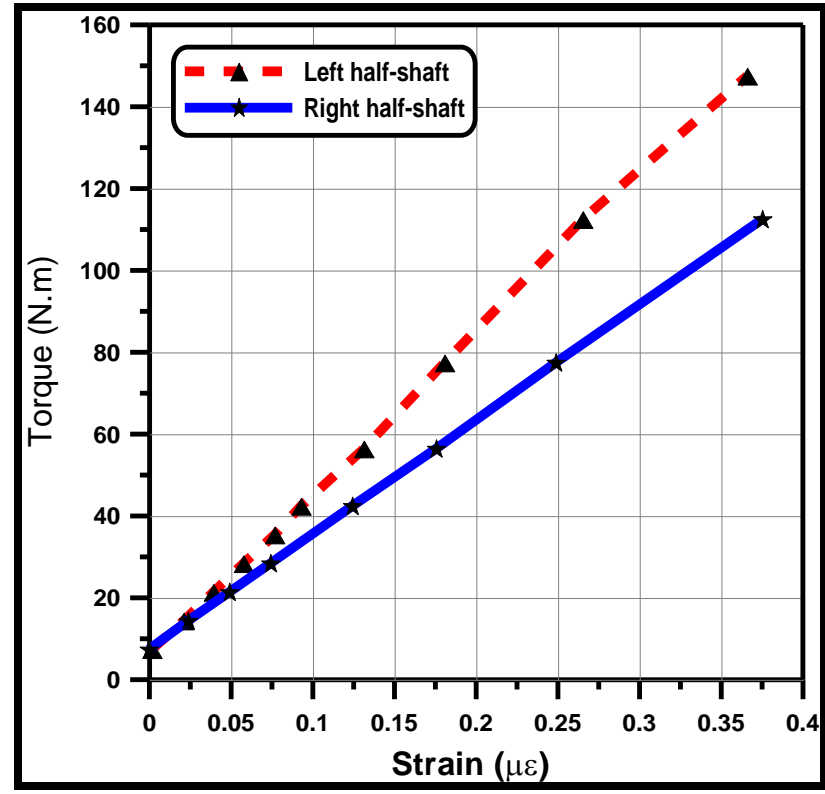

Fig. 7. Strain Gauge Calibration

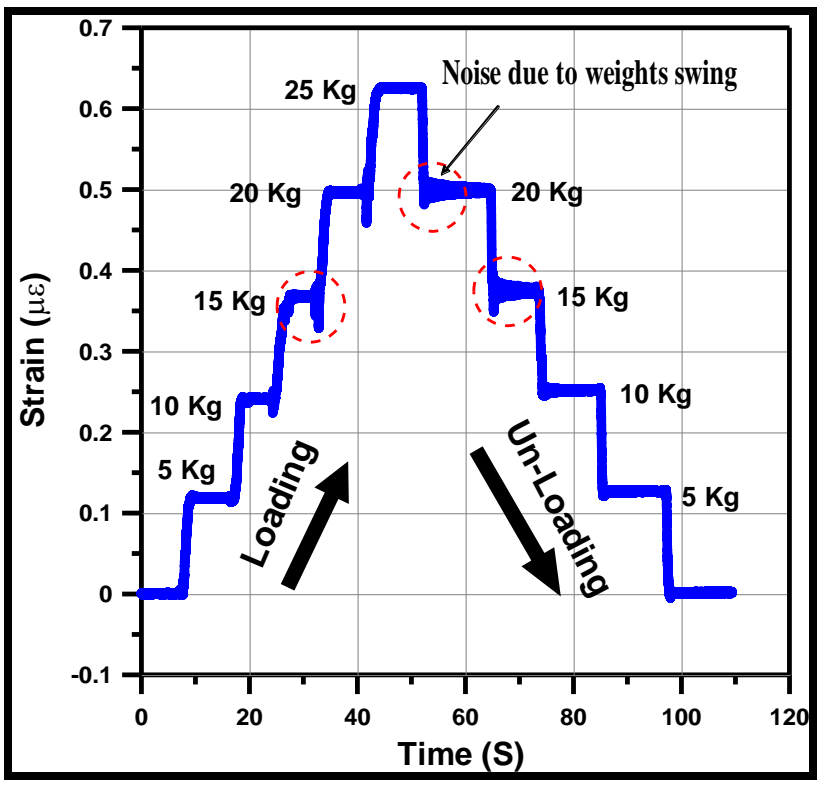

Fig. 9. Full Cycle Hysteresis Test

\section{Basic Control Strategy}

Consider a rear wheel drive vehicle negotiating a turn of radius (R), for free rolling conditions, the maximum rear wheel speed difference during cornering can be calculated as shown in Fig. 10. Assuming a limiting lateral acceleration of $1 \mathrm{~g}$, the maximum angular velocity at the vehicle centerline $(\beta)$ can be calculated as follows [16]:

lateral Acceleration $(\mathrm{g})=\frac{U^{2}}{R}=\frac{(\beta \cdot R)^{2}}{R} \Rightarrow \beta=\frac{\sqrt{\mathrm{g} \cdot R}}{R}=\sqrt{\frac{g}{R}}$

The wheel center velocities are then given by: 
$U_{L}=\left(R+\frac{t}{2}\right) \cdot \beta \quad, \quad U_{R}=\left(R-\frac{t}{2}\right) \cdot \beta$

The rotational wheel speed difference is therefore:

$\Delta \Omega=\Omega_{L}-\Omega_{R}=\frac{U_{L}}{r}-\frac{U_{R}}{r}=\frac{t}{r} \cdot \sqrt{\frac{g}{R}}$

With a given typical SUV vehicle parameters (wheel track, $\mathrm{t}=1.610 \mathrm{~m}$, and tire rolling radius, $\mathrm{r}=0.346 \mathrm{~m}$ ) and assuming a minimum turning radius of $10 \mathrm{~m}$, the maximum wheel speed difference that may be generated under free rolling conditions is unlikely to exceed 5 $\mathrm{rad} / \mathrm{s}$. Higher wheels rotational speed differences are only possible during traction or braking on $\mu$-split road conditions resulting in excessive wheels slipping or skidding.

The basic operation of the control system is based upon speed sensors which are used to measure the rotation of both left and right half-shafts of the drive axle. Corrective action will be initiated by the control system only if the wheels differential speed exceeds $5 \mathrm{rad} / \mathrm{s}$. The control unit regulates the applied current of the DC motor of the actuator via a double relays board (5 V control signal - 10 amperes -28 volts). Consequently, the direction of the DC motor can be controlled to perform both the engagement and disengagement process. Furthermore, a pulse width modulation technique is followed to control the strength of the engagement force and therefore the frictional clutch pack torque as shown in Fig. 11.

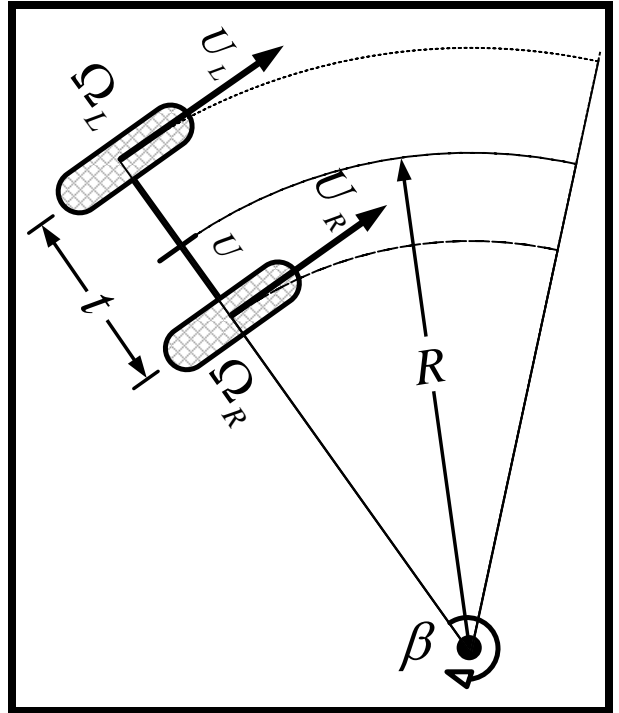

Fig. 10. Vehicle Rear Axle during Cornering

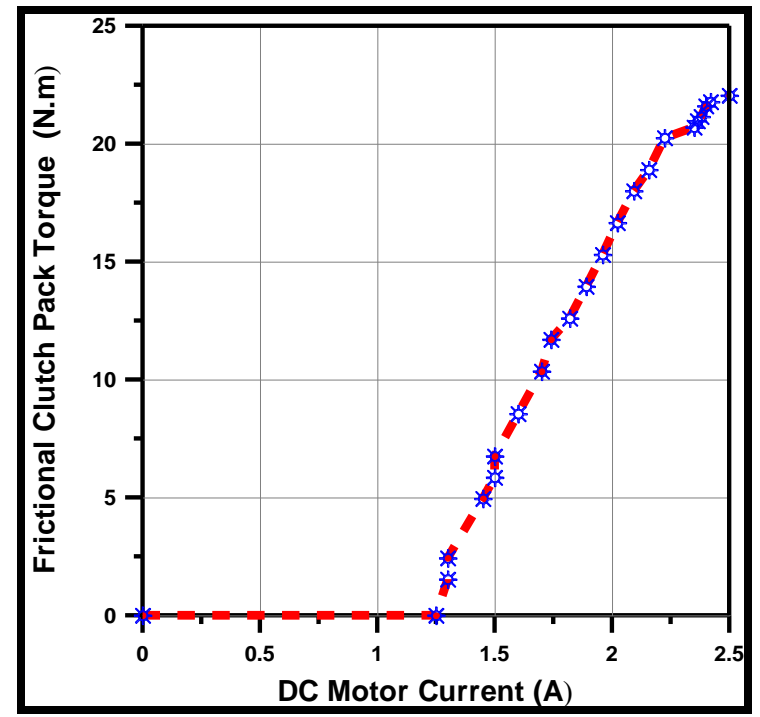

Fig. 11. Frictional Torque Characteristics

\section{Results and Analysis}

It is commonly recognized that, the road friction plays an important role in regulating the tire forces in both longitudinal and lateral directions. When the friction significantly differs between the left and the right wheels, the vehicle will be subjected to loss both the traction and direction stability. Higher rotational speed difference between the left and right wheels (> $5 \mathrm{rad} / \mathrm{s}$ ) is referred to an excessive tire slip during traction or tire skid during braking on such roads. According to the Newton's second law for rotational dynamics, the wheel angular 
acceleration and therefore the angular velocity are resulting from the wheel torque balance. Consequently, regulating the driving torque among wheels/ axles plays a crucial influence on controlling the wheels slippage. In order to measure the ability of the proposed device to control the torque distribution across the open differential, it is necessary to represent the condition of $\mu$-split road. This is carried out by applying different braking torque to the left and right wheels such that higher braking torque represents a good adhesion side and vice versa. It should be noted that, while in this work only one torque control device is applied, in a typical vehicle application, two devices will be employed.

The mathematical representation of the torque balance across the driving axles is introduced as shown in Fig. 12 which can be used as a guide to explain the measured results. For simplicity, the given equations are calculated during steady state conditions at which the inertial moments of the rotating parts can be neglected.

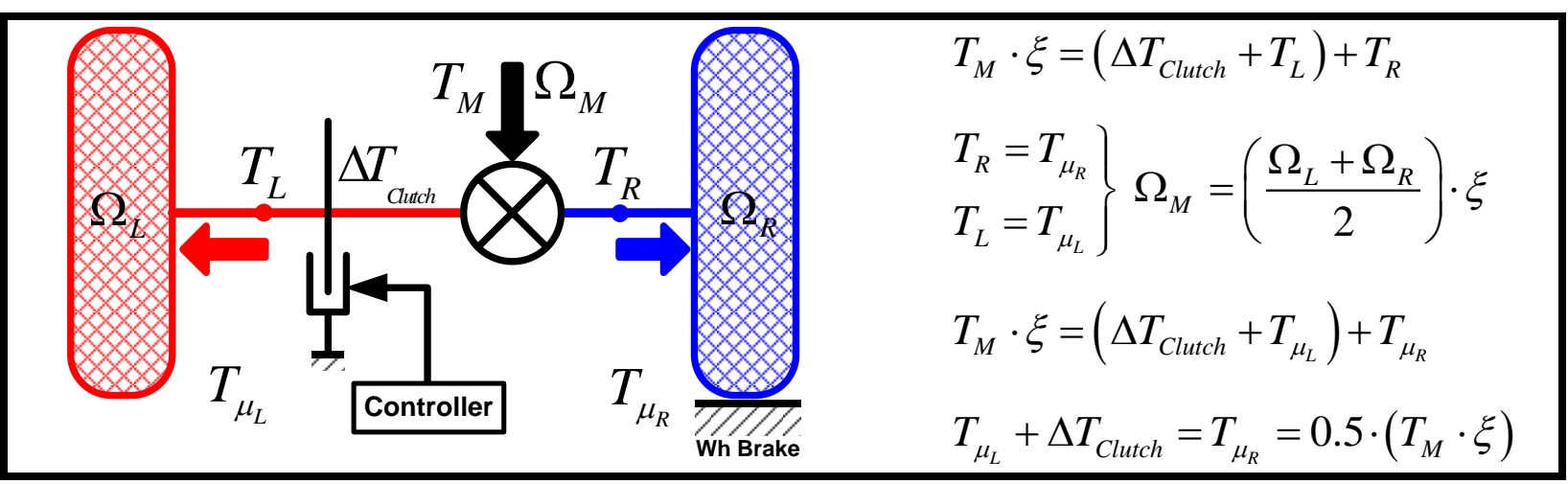

Fig. 12. The Mathematical Model of the Torque Distribution

The experiments are carried out during four different conditions and their results are illustrated in Figs 13 to 16. For each condition, both the rotational speed and driving torque of each half-shaft are measured. Initially, the AC electric motor drives the axle at suitable speed then the brakes are applied to both left and right wheels such that more braking torque is applied at the right wheel to generate rotational speed difference of approximately $250 \mathrm{rpm}$ ( $25 \mathrm{rad} / \mathrm{s})$. A speed controller is employed along with the motor to maintain the differential speed between the wheels constant.

The first experiment is carried out without activating the torque device $\left(\Delta T_{\text {Clutch }}=0\right)$ as shown in Fig. 13. It is obvious that, while there is a speed difference between both half-shafts, the measured torques at the rear-end of each half-shaft are approximately equal $\left(T_{\mu_{L}}=T_{\mu_{R}}\right)$. The slight difference between them can be referred to the difference in length of both shafts and also due to friction in the differentials. The obtained results agree with the characteristics of open differential which distributes driving torque evenly and limited by the lowest side of adhesion.

Similarly, the second experiment is carried out while the torque controlling device is activated. The method of activation is performed such that the maximum excitation current is instantaneously applied to the controlled DC motor resulting in an impulsive locking of the clutch pack. The introduction of the frictional clutch pack torque $\left(\Delta T_{\text {Clutch }}\right)$ results in an equal torque rise in the right side $\left(T_{\mu_{R}}\right)$ as shown in Fig. 14. On the other hand, the wheel speed difference is reduced and approximately both wheels are driven at close speed similar to that 
resulting from the mechanically locked differential. It should be noted that, the sudden increase in the torque of right half-shaft is accompanied with a little reduction of that in the left half-shaft; this effect may be referred to the motor controller which attempts to keep constant AC motor speed.

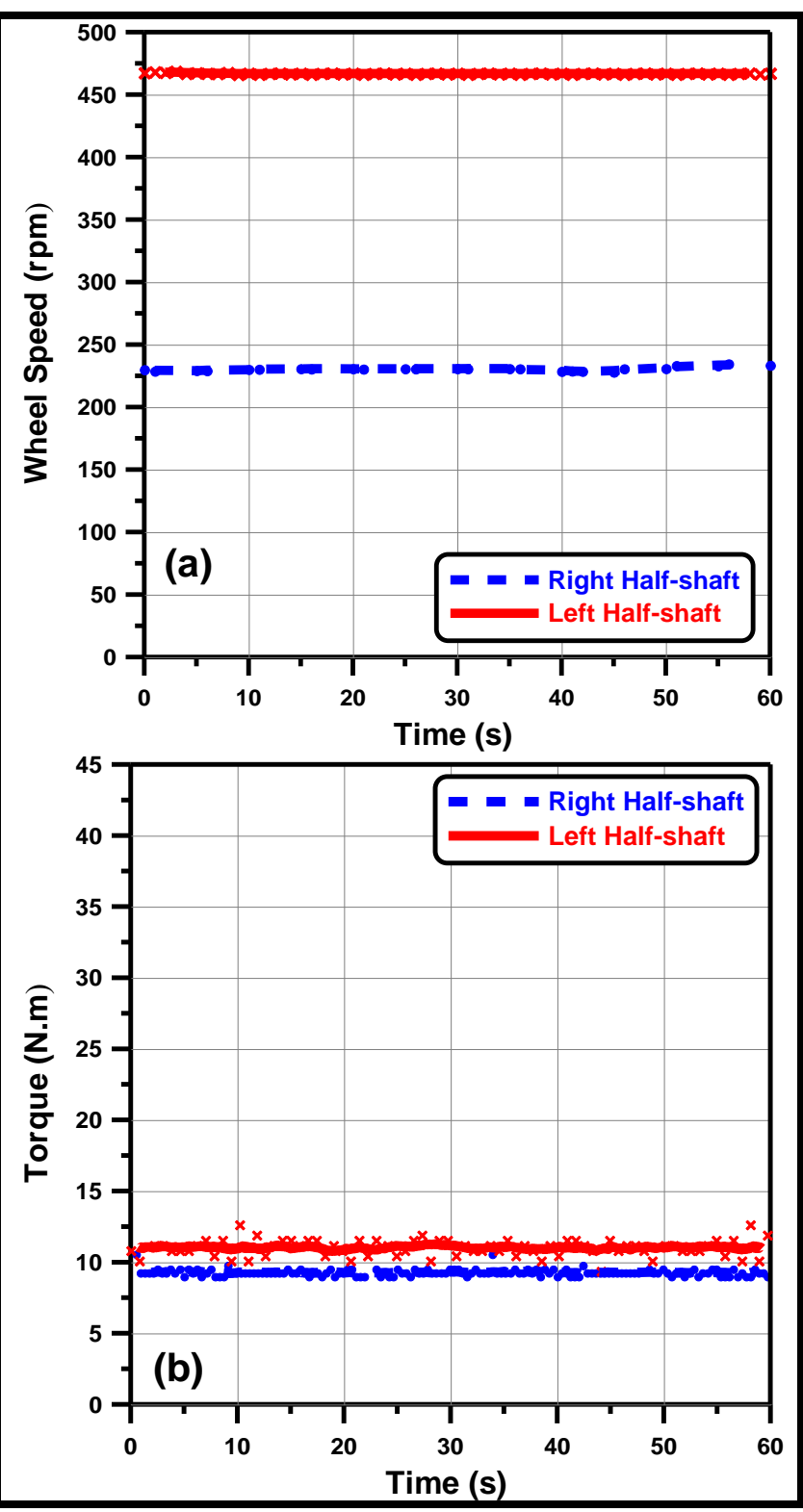

Fig. 13. Torque - Speed Characteristics without Activating the Torque Device
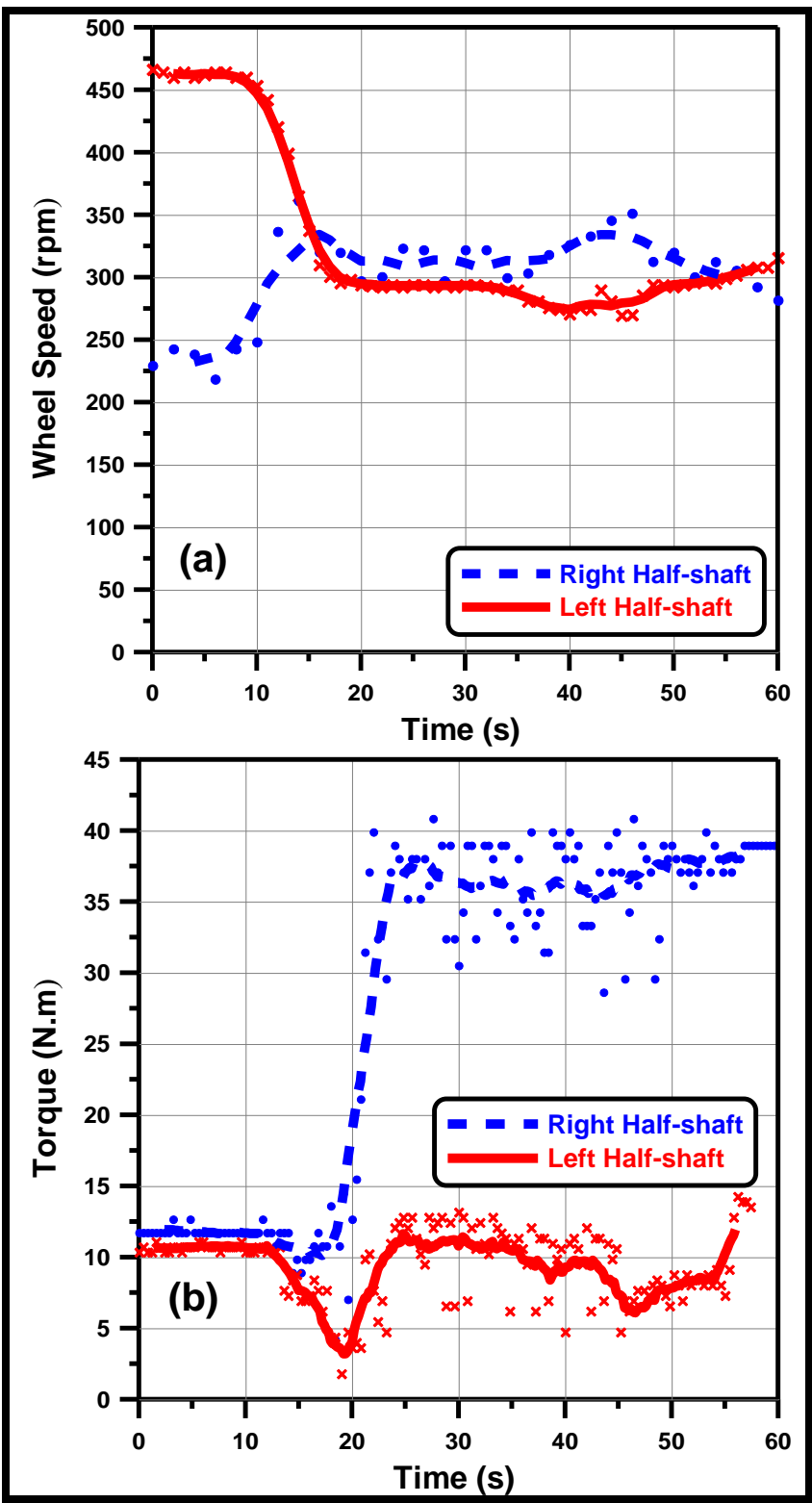

Fig. 14. Torque - Speed Characteristics with Activating the Torque Device

Since the maximum frictional torque which is transmitted by the clutch pack unit $\left(T_{F \max }\right)$ is proportional to the normal force applied to its plates $(F)$, as given in equation (1), therefore it would be possible to regulate this torque by modulating the current supplied to the DC motor. For this purpose another experiment is carried out through which a pulse-width modulation (PWM) or pulse-duration modulation (PDM) technique is employed for controlling the average value of the current fed to the DC motor. The longer the supplied current is on compared to the off periods, the higher the axial force and hence the frictional torque is. Fig. 15 depicts the torque and speed characteristics for $82.5 \%$ duty cycle. It can be noticed that, 
the torque achieves its maximum limit through a smoother and longer rising time. This effect would permit the possibility to accurately control the clutch pack fractional torque.

For further improvement of controlling the clutch pack fractional torque, a stepper motor is experienced. While it takes longer time to achieve the desirable control, Fig. 16 demonstrates that the wheel rotational speed come into closer through smoother and prolonged process. On the other hand, the biased torque can be obtained in the form of steps. The obtained result shows that it would be possible to control the biased torque by controlling the axial force given by the stepper motor.

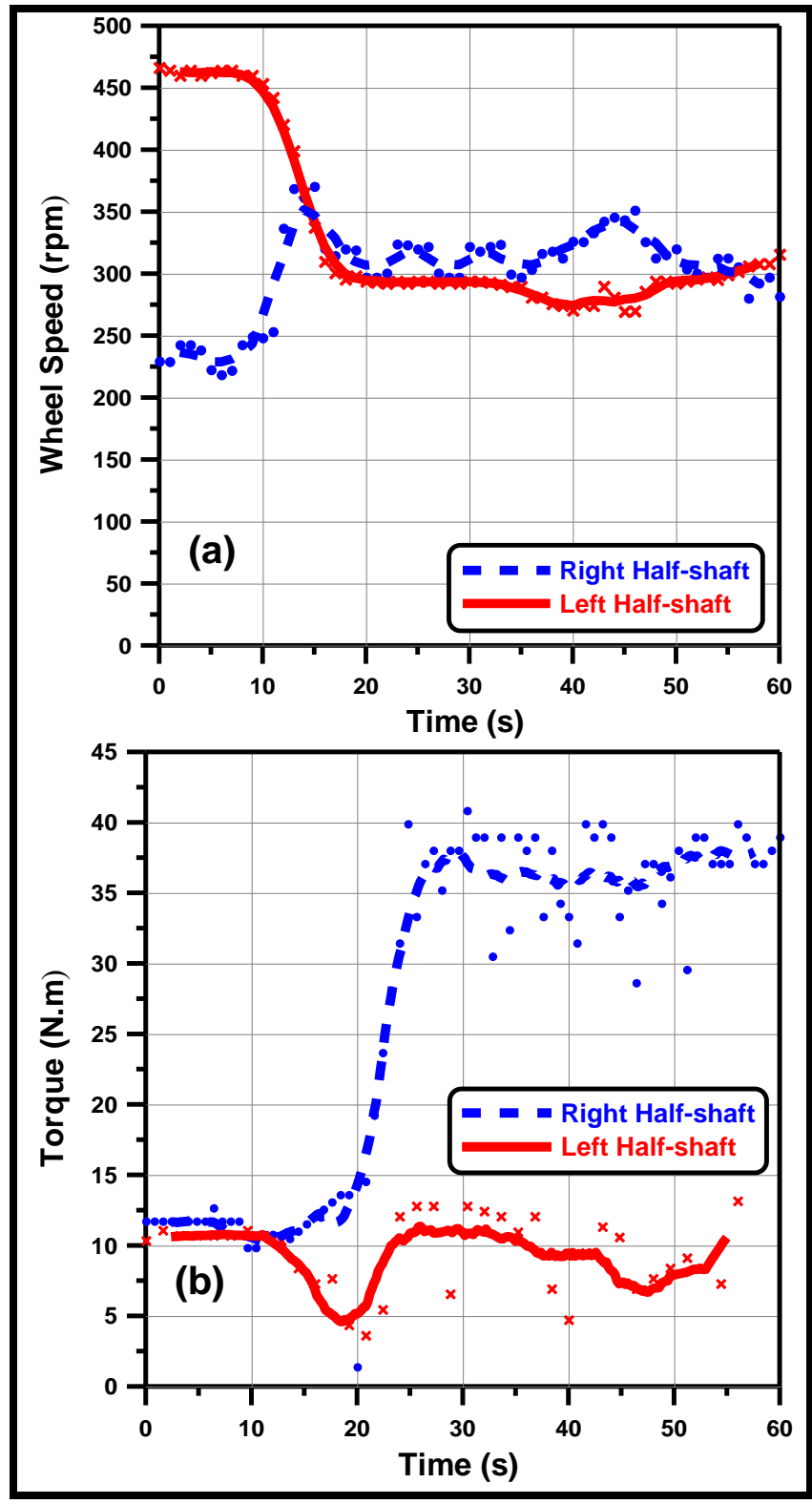

Fig. 15. Torque - Speed Characteristics with Activation through PWM of the DC Motor
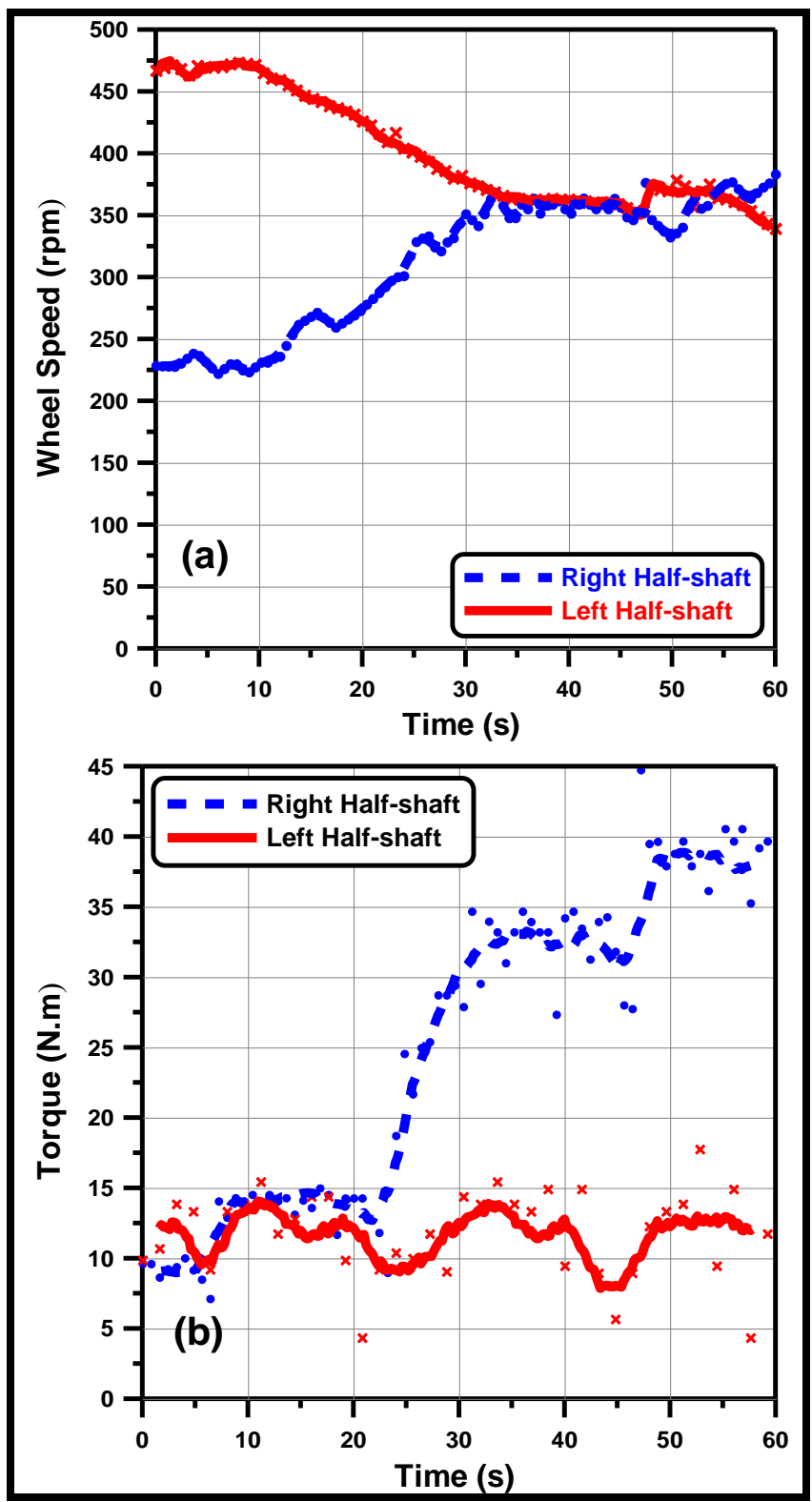

Fig. 16. Torque - Speed Characteristics with Activation through the Stepper Motor 


\section{Conclusion}

A comprehensive torque-vectoring device is developed in order to control the excessive tire slippage during straight forward maneuvers which in turn improve the overall traction potential. According to the difference in rotational speed of the differential output shafts, the presented device can bias more driving torque to the wheel with more frictional level. Special test rig is developed to investigate the control characteristics of the biasing torque. The obtained results prove the applicability of the proposed device to control the slip and therefore regulate the distributed torque across the output shafts of open differential.

\section{Acknowledgements}

The authors wish to express their sincere gratitude to Dr. Yasser ElShaer, the Department of Mechanical Design and Production, MTC, for his valuable support concerning the installation of the strain gauge and during the torque measurement.

\section{References}

[1] Sharaf, A.M., Mavros, G., Rahnejat, H. and King, P.D., "Multi-Physics Modelling Approach In All Terrain Vehicle Longitudinal Dynamics", Proceedings of IMECE06, ASME International Mechanical Engineering Congress and Exposition, November 510, 2006, Chicago, Illinois USA.

[2] Sharaf, A.M., Rahnejat, H. and King, P.D., "Analysis of Handling Characteristics of All-Wheel Drive Off-Road Vehicles", Int. J. Heavy Vehicle Systems, Vol. 15, No. 1, 2008, pp.89-106.

[3] Mohan, S.K. and Williams, R.C., "A Survey of 4WD Traction Control Systems and Strategies", SAE Technical Paper Series No. 952644, 1995, pp. 59-76.

[4] Liu, C., Monkaba, V., Tan, H., McKenzie, C., Lee, H. and Suo, S., "Driveline TorqueBias-Management Modeling for Vehicle Stability Control", SAE Technical Paper Series No. 2002-01-1584, 2002.

[5] Huchtkoetter, H. and Gassmann, T., "Vehicle Dynamics and Torque Management Devices", SAE Technical Paper Series No. 2004-01-1058, 2004.

[6] Sharaf, A.M., Mavros, G., Rahnejat, H., King, P.D. and Mohan, S.K., "Optimization of AWD Off-Road Vehicles Performance using Visco-Lock Devices", Int. J. Heavy Vehicle Systems, Vol. 15, Nos. 2/3/4, 2008.

[7] Kinsey, J., "The Advantages of an Electronically Controlled Limited Slip Differential", SAE Technical Paper Series No. 2004-1-0861, 2004.

[8] Park, J. and Kroppe, W., "Dana Torque Vectoring Differential Dynamic Trak", SAE Technical Paper Series No. 2004-01-2053 (P-386), 2004.

[9] Park, J., Dutkiewicz, J. and Cooper, K., "Simulation and Control of Dana's Active Limited-Slip Differential e-Diff”, SAE Technical Paper Series No. 2005-1-0409, 2005.

[10] Gassmann, T. and Barlage, J.A., "Electronic Torque Manager (ETM®): An Adaptive Torque Management System”, SAE Technical Paper Series No. 2004-01-0866, 2004.

[11] Wheals, J.C., Baker, H., Ramsey, K. and Turne, W., "Torque Vectoring AWD Driveline: Design, Simulation, Capabilities and Control", SAE Technical Paper Series No 2004-01-0863, 2004.

[12] Jonathan C. Wheals et al. "Torque Vectoring Driveline: SUV-based Demonstrator and Practical Actuation Technologies", SAE Technical Paper Series No. 2005-1-0553, 2005 
[13] Hancock, M., Williams, R., Fina, E. and Best, M., "Yaw Motion Control via Active Differentials", Transactions of the Institute of Measurement and Control, vol. 29, no. 2, 2007, pp. 137-157.

[14] Sawase, K. and Ushiroda, Y., "Improvement of Vehicle Dynamics by Right-and-left Torque Vectoring System in Various Drivetrains", Mitsubishi Technical Review, vol. 20, 2008, pp. 14-20.

[15] Ikushima, Y., and Sawase, K., "A Study on the Effects of the Active Yaw Moment Control", SAE Technical Paper Series No. 950303, 1995.

[16] Hancock, M.J., Williams, R.A., Gordon, T.J. and Best, M.C., "A Comparison of Braking and Differential Control of Road Vehicle Yaw-sideslip Dynamics", Proceedings of the Institution of Mechanical Engineers, Part D: Journal of Automobile Engineering, 219(3), 2005, pp.309-327. 